\title{
Variable initial swing side and prolonged double limb support represent abnormalities of the first three steps of gait initiation in patients with Parkinson's disease with freezing of gait
}

\author{
Yohei Okada ${ }^{1,2}$, Takahiko Fukumoto ${ }^{2}$, Katsuhiko Takatori ${ }^{2}$, Koji Nagino ${ }^{3}$ and Koichi Hiraoka ${ }^{4}$ \\ 1 Graduate School of Comprehensive Rehabilitation, Osaka Prefecture University, Osaka, Japan \\ ${ }^{2}$ Faculty of Health Science, Kio University, Nara, Japan \\ ${ }^{3}$ Faculty of Allied Health Sciences, Division of Physical Therapy, Department of Rehabilitation Sciences, Kansai University of Welfare Sciences, Osaka, Japan \\ ${ }^{4}$ School of Comprehensive Rehabilitation, Osaka Prefecture University, Osaka, Japan
}

\author{
Edited by: \\ Ryuji Kaji, Tokushima University \\ Hospital, Japan \\ Reviewed by: \\ Pratap Chand, Saint Louis University, \\ USA \\ Beom S. Jeon, Seoul National \\ University Hospital, South Korea \\ ${ }^{*}$ Correspondence: \\ Koichi Hiraoka, School of \\ Comprehensive Rehabilitation, Osaka \\ Prefecture University, Habikino 3-7-30, \\ Habikino city, Osaka 583-8555, Japan. \\ e-mail: hiraoka@rehab.osakafu-u.ac.jp
}

This study investigated abnormalities of the first three steps of gait initiation in patients with Parkinson's disease without freezing of gait (PD - FOG) and investigated which abnormalities are related to FOG. Seven PD - FOG and seven age-matched healthy controls performed self-generated or cue-triggered gait initiation. Data for PD patients with FOG (PD + FOG) were cited from a previous study using a procedure similar to that used in the present study. Gait initiation was abnormal, and external cue normalized some abnormalities in PD - FOG. The initial swing side was fairly consistent among the trials in both PD - FOG and in healthy controls, although the initial swing side was inconsistent in PD + FOG. The duration of the first double limb support (DLS) was the only parameter that depends on FOG severity and that was abnormal in PD + FOG but was not abnormal in PD - FOG. The variability of the initial swing side and prolonged first DLS are abnormalities specifically related to FOG.

Keywords: Parkinson's disease, gait initiation, freezing of gait

\section{INTRODUCTION}

Gait initiation is impaired in patients with Parkinson's disease (PD; Crenna et al., 1990; Gantchev et al., 1996; Burleigh-Jacobs et al., 1997; Rosin et al., 1997; Halliday et al., 1998; Dibble et al., 2004; Hiraoka et al., 2006; Carpinella et al., 2007; Hass et al., 2008; Okada et al., 2011). In particular, abnormality is prominent during the postural phase, defined as the period between the onset of the weight shifting to the initial swing side and the onset of the heel off in the initial swing limb. A prolonged postural phase (Gantchev et al., 1996; Rosin et al., 1997; Halliday et al., 1998), decreased displacement of the center of pressure (COP; Halliday et al., 1998; Dibble et al., 2004; Carpinella et al., 2007; Hass et al., 2008; Okada et al., 2011), decreased depression of motoneuron pool excitability of the soleus muscle (Hiraoka et al., 2006), and abnormal electromyographic activities in the leg muscles (Gantchev et al., 1996) have been observed in this phase.

The transition phase between gait initiation and steady-state gait is also abnormal in PD patients with freezing of gait (PD + FOG; Okada et al., 2011). In this previous study, the first three steps of gait initiation were investigated because two or three steps are necessary for the transition from gait initiation to steadystate gait in healthy humans (Mann et al., 1979). Trial-to-trial variability of the initial swing side of gait initiation was observed, indicating a variable motor pattern during gait initiation. The COP peak displacement and the heel contact position deviated toward the initial swing side during the first step of gait initiation, indicating excessive weight shifting toward the initial swing side in this phase. Medial deviation of the COP from the heel contact position was observed during the first two steps of gait initiation, indicating that a certain amount of weight remains on the tracing leg contralateral to the heel contact side.

Such abnormalities of gait initiation may be specifically related to FOG, during which patients suddenly become unable to start walking or to continue moving forward. PD patients experience FOG during gait initiation with a prevalence of $58 \%$ of $\mathrm{PD}$ patients in the "OFF" state and 5\% of those in the "ON" state (Schaafsma et al., 2003). Abnormalities have been found in PD + FOG during steady-state gait or during treadmill walking (Nieuwboer et al., 2001, 2004; Hausdorff et al., 2003; Plotnik et al., 2005, 2008; Iansek et al., 2006; Chee et al., 2009; Nanhoe-Mahabier et al., 2011). Stride time variability in $\mathrm{PD}+\mathrm{FOG}$ is higher than that in $\mathrm{PD}$ patients without FOG (PD - FOG) during steady-state gait (Hausdorff et al., 2003). Step length in PD + FOG is shorter than that in PD - FOG during treadmill walking (Nanhoe-Mahabier et al., 2011) and during steady-state gait (Chee et al., 2009). Step velocity in PD + FOG is less than that in PD - FOG during steady-state gait (Chee et al., 2009). Asymmetry and bilateral incoordination during steady-state gait are present in PD + FOG (Plotnik et al., $2005,2008)$. Step length progressively decreases as the number of steps increases during steady-state gait in PD + FOG (Iansek et al., 2006; Chee et al., 2009). Before the occurrence of freezing during steady-state gait, stride length and gait velocity decrease, the 
double limb support (DLS) phase and cadence increase (Nieuwboer et al., 2001), and premature onset of tibialis anterior and gastrocnemius muscle activities occurs (Nieuwboer et al., 2004).

However, the findings concerning abnormalities during the postural phase are derived from the whole population of $\mathrm{PD}$ patients including both PD + FOG and PD - FOG (Crenna et al., 1990; Gantchev et al., 1996; Burleigh-Jacobs et al., 1997; Rosin et al., 1997; Halliday et al., 1998; Dibble et al., 2004; Hiraoka et al., 2006; Carpinella et al., 2007; Hass et al., 2008). All of the previous studies investigating abnormalities between PD + FOG and PD - FOG are derived from steady-state gait or treadmill walking (Hausdorff et al., 2003; Plotnik et al., 2005, 2008; Chee et al., 2009; Nanhoe-Mahabier et al., 2011). Previous findings concerning abnormalities of the first three steps of gait initiation are derived only from PF + FOG (Okada et al., 2011). Accordingly, there has been no study comparing abnormalities of gait initiation between PD + FOG and PD - FOG. FOG most frequently occurs at turns or during gait initiation (Schaafsma et al., 2003), indicating that FOG may be more prominent during gait initiation than during steady-state gait. Therefore, the present study investigated abnormalities in the first three steps of gait initiation in PD - FOG and healthy controls, and compared the abnormalities in PD - FOG with those in PD + FOG that were reported in a previous study conducted using the same apparatus and procedure (Okada et al., 2011). The present study using "historical control data" from a group of patients who were observed at some time in the past is based on an experimental design called " historical control design” (Sacks et al., 1982; Lee and Tseng, 2001) which has been applied for clinical trials (Cupp et al., 2004; Meng et al., 2009).

Medial deviation of the COP from the first heel contact position during gait initiation was found to be abnormal in PD + FOG, and was dependent on FOG severity, implying that medial deviation of the COP from the first heel contact position may be specifically related to FOG (Okada et al., 2011). However, it is impossible to rule out the possibility that FOG severity-dependent medial deviation of the COP from the first heel contact position may have originated due to the severity of the disease, because FOG severity partially depends on disease severity. PD + FOG have significantly severe unified Parkinson's disease rating scale (UPDRS) motor scores, severe Hoehn and Yahr (H-Y) stages, and longer disease duration as compared to PD - FOG (Chee et al., 2009). FOG is correlated with UPDRS motor score (Giladi et al., 2000, 2009) and duration of the disease (Lamberti et al., 1997). Therefore, comparing abnormalities in gait initiation between PD + FOG and PD - FOG groups with equal disease severity is needed to investigate which abnormalities of gait initiation are specifically related to FOG. In the present study, the difference in disease severity between PD + FOG and PD - FOG was statistically tested to confirm whether the PD patients included in the present study satisfied the condition of equal disease severity.

External cues function to improve abnormalities in the postural phase of gait initiation in PD patients by reducing the duration of the postural phase (Burleigh-Jacobs et al., 1997), increasing force production (Burleigh-Jacobs et al., 1997), increasing COP displacement during the postural phase (Dibble et al., 2004), and decreasing DLS (Dibble et al., 2004). Furthermore, the tibialis anterior muscle burst and soleus H-reflex depression during the postural phase of cue-triggered gait initiation were larger than those during the postural phase of self-generated gait initiation (Hiraoka et al., 2006). However, it is not fully understood whether external cues bring about improvements in the abnormalities that occur during the transition phase between gait initiation and steady-state gait in PD patients. Therefore, abnormalities in the first three steps of gait initiation were compared between self-generated and cue-triggered gait initiation.

\section{MATERIALS AND METHODS \\ SUBJECTS}

Seven PD - FOG (six males and one female), aged from 58 to 78 years, and seven age-matched healthy controls (five males and two females), aged from 61 to 73 years, participated (Table 1). An unpaired $t$-test revealed no significant difference in age between the groups. PD - FOG subjects were able to walk independently at least $10 \mathrm{~m}$ without assistive devices. PD patients with a freezing of gait questionnaire (FOGQ) item 3 score $=0$, which meant that they had not experienced the feeling of their feet getting glued to the ground in any situation (Giladi et al., 2000), were included in the experiment. FOGQ item 3 score $=0$ indicated that all the PD patients included in the present study did not suffer FOG according to the criteria previously defined (Giladi et al., 2009). The FOGQ total score ranged from 0 to 12 . The score on the footedness subscale of the laterality preference inventory (Coren, 1993) was 4 in all of healthy controls and PD - FOG patients, except in one PD - FOG patient whose score was 0 . Based on the cut-off criteria devised by Coren, all the subjects who participated in the present study were classified as right-footers except one PD - FOG patient who was classified as a left-footer. All the PD - FOG patients were being treated with stable doses of antiparkinsonian medications. The total daily levodopa equivalent dose (LED) ranged from 300 to $600 \mathrm{mg} /$ day. The PD - FOG patients were not being treated with $\mathrm{MAOB}$ inhibitors such as selegiline or rasagiline, and with norepinephrine and dopamine reuptake inhibitors such as methyl phenidate (Ritalin). All the experiments were conducted while $\mathrm{PD}$ - FOG subjects were in the "ON" state. All the subjects gave written informed consent for study participation in accordance with the Helsinki Declaration. The experiment was approved by the ethical committee of Osaka Prefecture University.

Additionally, historical control data were cited from a previous study; the findings regarding self-generated gait initiation in PD + FOG were also cited from a previous study (Okada et al., 2011) conducted using the same apparatus and procedure as the present study in order to compare the abnormalities of gait initiation between PD + FOG and PD - FOG. Ten PD patients (six males and four females), aged from 63 to 88 years, were included in the previous study. H-Y stage ranged from 2 to 4 , and UPDRS motor score ranged from 5 to 24. A Mann-Whitney test revealed no significant differences in age, $\mathrm{H}-\mathrm{Y}$ stage, and UPDRS motor score between the PD + FOG and PD - FOG. Total FOGQ scores ranged from 3 to 22, and the scores on FOGQ item 5 ranged from 0 to 3 . The FOGQ item 3 scores ranged from 1 to 3 , indicating that $\mathrm{PD}$ patients in the previous study can be categorized as PD + FOG according to the cut-off criteria (Giladi et al., 2009). LED ranged from 150 to $500 \mathrm{mg} /$ day. An unpaired $t$-test revealed that LED 
Table 1 | Characteristics of PD patients.

\begin{tabular}{|c|c|c|c|c|c|c|c|c|}
\hline Subject & Age (years) & Sex & $H$ and $Y$ stage & UPDRS motor & LED (mg/day) & FOGQ total & FOGQ-item3 & LPI footedness \\
\hline 1 & 63 & $\mathrm{M}$ & 2 & 21 & 400 & 0 & 0 & 4 \\
\hline 2 & 78 & $\mathrm{M}$ & 2 & 7 & 300 & 7 & 0 & 0 \\
\hline 3 & 58 & $M$ & 3 & 7 & 600 & 12 & 0 & 4 \\
\hline 4 & 68 & $\mathrm{~F}$ & 3 & 20 & 400 & 4 & 0 & 4 \\
\hline 5 & 74 & $\mathrm{M}$ & 2 & 14 & 600 & 3 & 0 & 4 \\
\hline 6 & 63 & $\mathrm{M}$ & 3 & 20 & 600 & 4 & 0 & 4 \\
\hline 7 & 71 & $\mathrm{M}$ & 1 & 5 & 400 & 4 & 0 & 4 \\
\hline
\end{tabular}

M, male; F, female; LED, levodopa equivalent dose; FOGQ, freezing of gait questionnaire; LPI, lateral preference inventory.

in $\mathrm{PD}-\mathrm{FOG}$ was significantly higher than that in $\mathrm{PD}+\mathrm{FOG}$ $(P<0.05)$.

\section{APPARATUS}

Gait initiation was performed on a force platform (The FDMSystem 2, Zebris Medical GmbH, Isny, Germany) placed on the first $2.18 \mathrm{~m}$ of a 9-m-long walkway. The length of the platform was expected to be sufficient to record three steps of gait initiation. The width of the platform was $0.6 \mathrm{~m}$. This platform recorded the vertical pressure on the surface of the force platform each $0.75 \mathrm{~cm}^{2}$ at a sampling rate of $100 \mathrm{~Hz}$. COP, heel contact positions, and spatiotemporal parameters were estimated from the vertical pressures.

\section{PROCEDURE}

First, the subjects stood at the beginning of the walkway. The subjects gazed at a target point located at the approximate center of the walkway but $4 \mathrm{~m}$ beyond the end of the walkway. In self-generated gait initiation, the subjects initiated walking at their own pace, while in cue-triggered gait initiation, subjects initiated walking with an auditory start cue generated from a speaker. Several practice trials were performed before the experimental trials were begun. The two types of gait initiation were performed in a random order. The experimental trials were repeated until the subjects successfully initiated walking seven times for each type of gait initiation.

\section{COP DISPLACEMENT}

The COP trajectory between the onset of the displacement of the COP beginning from quiet standing and the fourth toe off was analyzed (Figure 1). The beginning of the COP trajectory was defined as zero along the anteroposterior and mediolateral axes. A positive COP value indicates anterior displacement along the anteroposterior axis and deviation to the initial stance side in reference to zero along the mediolateral axis. The duration between the onset of COP displacement and the fourth toe off was defined as $100 \%$. Then, the COP was averaged for each $5 \%$ bin of the duration. That is, 20 mean COP data points were plotted between the onset of COP displacement and the fourth toe off.

The first COP peak displacement was defined as the COP most deviated to the initial swing side before the first heel off. The first COP peak displacement was identical to the end of the S1 period, which began with the start command and ended with the COP located in its most posterior and lateral position toward the initial swing side (Hass et al., 2004). The second COP peak displacement was defined as the first peak of the COP to the initial stance side after the first COP peak displacement. The third COP peak displacement was defined as the first peak of the COP to the initial swing side after the second COP peak displacement. The fourth COP peak displacement was defined as the first peak of the COP to the initial stance side after the third COP peak displacement.

\section{HEEL CONTACT POSITION}

The heel contact position along the anteroposterior axis was estimated from the position of the rear end of the heel. The heel contact position along the mediolateral axis was estimated from the position of the centerline of the heel. The amount of medial deviation of the COP from the heel contact position was also estimated. A positive value denoted medial deviation of the COP from the heel contact position, while a negative value denoted lateral deviation of the COP from the heel contact position.

\section{SPATIOTEMPORAL PARAMETERS}

The first step time was defined as the period between the onset of COP displacement and the first heel contact. The second step time was defined as the period between the first heel contact and the second heel contact, and the third step time was defined as the period between the second heel contact and the third heel contact. The single limb support (SLS) duration of each step was defined as the period between toe off and the next heel contact. The DLS duration was the period between the end of the SLS and the beginning of the next SLS. The DLS/Cycle ratio was defined as the DLS duration divided by the cycle comprising sum of the SLS and DLS durations. The step length was defined as the distance between the heel contact positions along the anteroposterior axis. The step width was defined as the distance between the heel contact positions along the mediolateral axis. The step velocity was expressed as the step length divided by the step time.

\section{STATISTICAL ANALYSIS}

Data were presented as the mean values and SE of the mean. Subjects who initiated walking with the same leg in all of the trials were considered to be subjects who initiated walking with a consistent initial swing side. The number of subjects who initiated walking with the same leg in all the trials was divided by the total number of subjects to estimate the proportion of total subjects who initiated walking with a consistent initial swing side. The difference in the two proportions was statistically tested. Unpaired $t$-tests or 


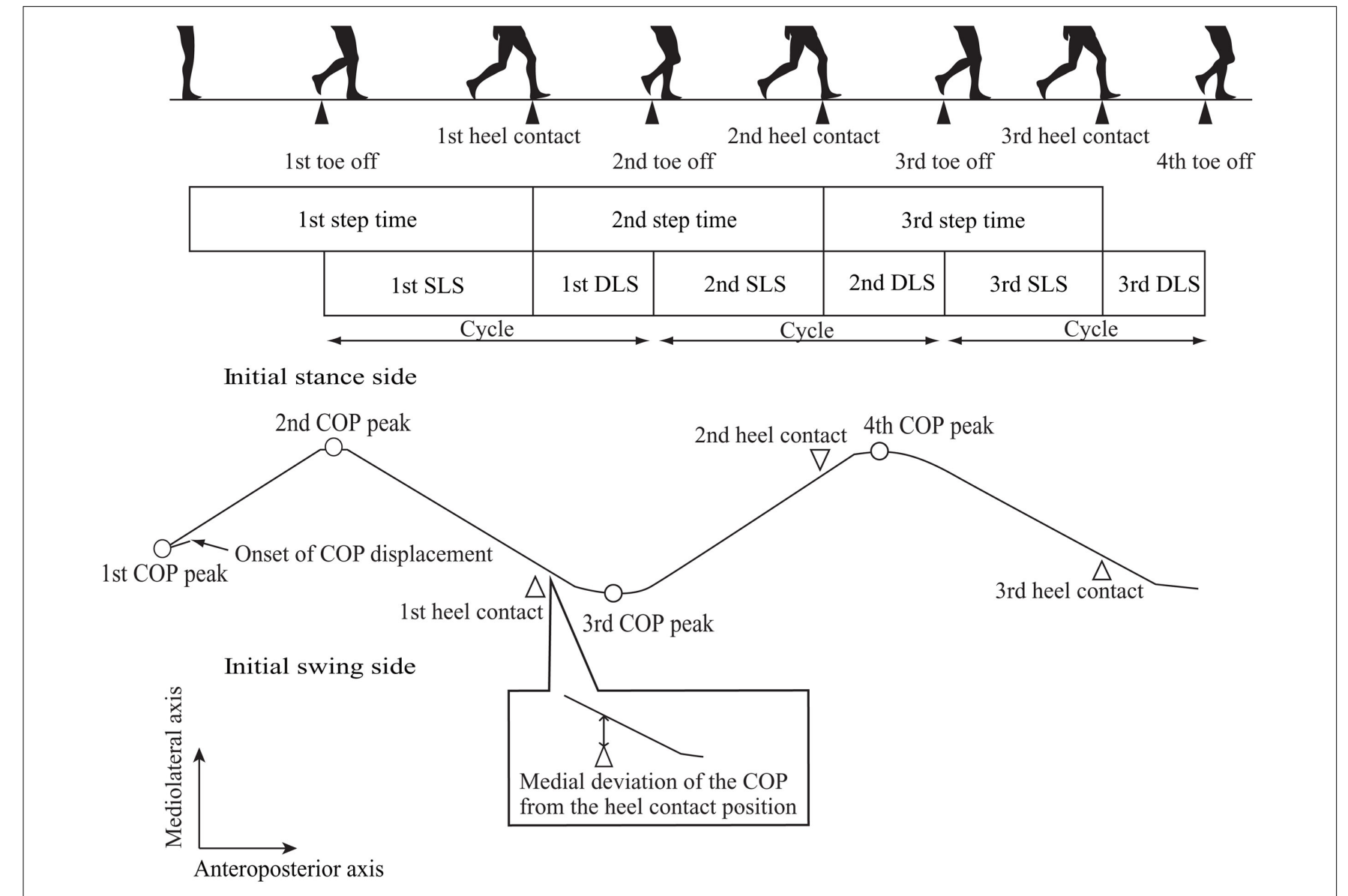

FIGURE 1 | Spatiotemporal and kinesiological parameters.

a Mann-Whitney test were conducted to examine the difference in the means between the groups. The alpha level was 0.05. Effect size (Thomas et al., 1986), the difference in means between the groups divided by the SD of the control group, was used to estimate the magnitude of the difference in means between PD + FOG and PD - FOG. The statistical significance levels of the Spearman's rank correlation coefficients for the parameters in PD + FOG were cited from the previous study (Okada et al., 2011).

\section{RESULTS \\ GENERAL FEATURES}

All the subjects were able to initiate walking without assistance. The first three steps were performed completely within the force platform in all trials by all subjects. Five healthy controls and three PD - FOG patients initiated gait with the preferred leg as classified by the laterality preference inventory score, but the other subjects initiated gait mostly with the non-preferred leg. The initial swing side was consistent throughout the trials in all subjects, except in one healthy control during self-generated gait initiation $(6 / 7=0.86$ during self-generated gait initiation and $7 / 7=1.00$ during cue-triggered gait initiation) and in one PD - FOG during self-generated and cue-triggered gait initiation $(6 / 7=0.86)$. There was no statistical difference in the proportion of the total number of subjects who initiated walking with the same leg in all trials between the groups during both self-generated and cue-triggered gait initiation.

\section{SPATIOTEMPORAL PARAMETERS}

The first step time in PD - FOG was significantly longer than that in healthy controls during self-generated gait initiation $(P<0.05)$, but it was not significantly different between the groups during cue-triggered gait initiation. The second and third step times were not significantly different between the groups during either selfgenerated or cue-triggered gait initiation (Table 2). Step length and step velocity in PD - FOG were significantly less than those in healthy controls during both self-generated and cue-triggered gait initiation $(P<0.05)$, except for the first step velocity during cue-triggered gait initiation. The step width was not significantly different between the groups during either self-generated or cuetriggered gait initiation. The DLS/Cycle ratios were not significantly different between the groups during either self-generated or cue-triggered gait initiation, except for the second DLS/Cycle ratio, which was significantly larger in PD - FOG as compared with healthy controls during self-generated gait initiation $(P<0.05)$.

\section{COP DISPLACEMENT}

The first COP peak displacement along the anteroposterior axis in $\mathrm{PD}-\mathrm{FOG}$ was significantly smaller than that in healthy 


\begin{tabular}{|c|c|c|c|c|c|c|}
\hline & \multicolumn{2}{|c|}{ Cue-triggered } & \multicolumn{3}{|c|}{ Self-generated } & \multirow[t]{2}{*}{ Effect size } \\
\hline & Healthy & PD - FOG & Healthy & PD - FOG & PD + FOG & \\
\hline Consistency of the initial swing side & $7 / 7$ & $6 / 7$ & $6 / 7$ & $6 / 7$ & $3 / 10^{*}$ & \\
\hline \multicolumn{7}{|l|}{ STEP LENGTH (cm) } \\
\hline $1 \mathrm{st}$ & $52.3(1.4)$ & $45.2(2.9)^{*}$ & $52.5(1.1)$ & $44.8(2.8)^{*}$ & $38.0(3.8)^{*^{\dagger}}$ & -0.93 \\
\hline 2nd & $55.4(0.8)$ & $46.5(2.4)^{*}$ & $55.5(0.9)$ & $45.8(2.9)^{*}$ & $40.1(3.6)^{*^{+}}$ & -0.75 \\
\hline $3 r d$ & $57.9(0.9)$ & $51.4(2.2)^{*}$ & $57.9(1.2)$ & $50.7(2.3)^{*}$ & $44.9(3.9)^{*^{\dagger}}$ & -0.96 \\
\hline \multicolumn{7}{|l|}{ STEP TIME (s) } \\
\hline $1 \mathrm{st}$ & $0.99(0.03)$ & $1.02(0.06)$ & $0.97(0.03)$ & $1.12(0.06)^{*}$ & $1.14(0.06)^{*}$ & 0.13 \\
\hline 2 nd & $0.61(0.01)$ & $0.59(0.02)$ & $0.63(0.02)$ & $0.61(0.02)$ & $0.63(0.03)$ & 0.38 \\
\hline $3 r d$ & $0.53(0.01)$ & $0.52(0.02)$ & $0.53(0.02)$ & $0.54(0.02)$ & $0.54(0.02)$ & 0.00 \\
\hline \multicolumn{7}{|l|}{ STEP WIDTH } \\
\hline $1 \mathrm{st}$ & $7.5(0.9)$ & $10.1(1.1)$ & $7.4(1.1)$ & $9.5(1.1)$ & $8.5(0.9)$ & -0.76 \\
\hline 2nd & $8.0(1.2)$ & $7.3(1.1)$ & $6.8(1.0)$ & $7.2(1.1)$ & $7.8(1.4)$ & 0.59 \\
\hline $3 r d$ & $6.2(1.0)$ & $7.6(1.3)$ & $5.8(0.9)$ & $7.7(1.4)$ & $7.8(1.3)$ & 0.15 \\
\hline \multicolumn{7}{|l|}{ STEP VELOCITY (m/min) } \\
\hline $1 \mathrm{st}$ & $32.0(1.3)$ & $27.6(2.9)$ & $33.1(1.1)$ & $24.8(1.8)^{*}$ & $22.0(2.4)^{*^{\dagger}}$ & -0.60 \\
\hline 2nd & $54.6(0.9)$ & $47.2(2.2)^{*}$ & $53.4(1.1)$ & $44.9(2.2)^{*}$ & $39.4(3.8)^{*^{+}}$ & -0.94 \\
\hline $3 r d$ & $66.3(1.0)$ & $59.0(4.1)^{*}$ & $65.5(1.6)$ & $56.9(2.0)^{*}$ & $50.3(4.1)^{*^{+}}$ & -1.25 \\
\hline \multicolumn{7}{|l|}{ DLS RATIO } \\
\hline $1 \mathrm{st}$ & $0.33(0.01)$ & $0.35(0.01)$ & $0.32(0.01)$ & $0.34(0.01)$ & $0.38(0.03)^{*^{\dagger}}$ & 1.91 \\
\hline 2nd & $0.25(0.01)$ & $0.28(0.02)$ & $0.25(0.01)$ & $0.28(0.01)^{*}$ & $0.31(0.02)^{*^{+}}$ & 0.88 \\
\hline $3 r d$ & $0.25(0.01)$ & $0.27(0.02)$ & $0.25(0.00)$ & $0.27(0.02)$ & $0.30(0.03)^{\dagger}$ & 0.74 \\
\hline
\end{tabular}

Mean (SE of mean).

Asterisks indicate significant difference between PD patients and Healthy controls.

Daggers indicate significant correlation between the parameter in PD+FOG and FOGQ item 5.

The data in PD+FOG were cited from the previous study (Okada et al., 2011).

controls during self-generated gait initiation $(P<0.05)$, although it was not significantly different between the groups during cuetriggered gait initiation (Figure 2; Table 3). The second COP peak displacement along the anteroposterior axis in PD-FOG was not significantly different between the groups during both selfgenerated and cue-triggered gait initiation (Figure 3; Table 3). The third and fourth COP peak displacements along the anteroposterior axis in PD - FOG were significantly posterior as compared to those in healthy controls during both self-generated and cue-triggered gait initiation $(P<0.05)$. The third COP peak displacement to the initial swing side along the mediolateral axis in PD - FOG was significantly larger than that in healthy controls during both self-generated and cue-triggered gait initiation $(P<0.05)$. However, no significant difference was observed in the first, second, and fourth COP peak displacements during either self-generated or cue-triggered gait initiation.

\section{HEEL CONTACT POSITION}

The first and third heel contact positions significantly deviated to the initial swing side as compared to those in healthy subjects during both self-generated and cue-triggered gait initiation $(P<0.05$; (Figure 3; Table 3). The second heel contact position in PD - FOG significantly deviated to the initial swing side in PD - FOG as compared with that in healthy controls during cue-triggered gait initiation $(P<0.05)$, but this position was not significantly different during self-generated gait initiation.

\section{MEDIAL DEVIATION OF THE COP FROM THE HEEL CONTACT POSITION}

The COP trajectory in PD - FOG passed medial to each heel contact position during the first step in comparison with healthy controls during both self-generated and cue-triggered gait initiation (Figure 3). The medial deviation of the COP from the first heel contact position was significantly larger than that in healthy controls during self-generated gait initiation $(P<0.05$; Table 3$)$. The medial deviations of the COP from the second or third heel contact position were not significantly different between the groups during self-generated gait initiation. The medial deviations of the COP from the heel contact position were not significantly different between groups during cue-triggered gait initiation.

\section{COMPARISON BETWEEN PD + FOG AND PD - FOG}

In addition to the data obtained from PD - FOG, the means and $\mathrm{SE}$ of the gait parameters during self-generated gait initiation in PD + FOG are cited from the previous study (Okada et al., 2011) and the effect sizes between PD + FOG and PD - FOG are shown in Tables 2 and 3. According to the data in the previous study, the initial swing side was consistent in all of the healthy controls $(10 / 10=1.00)$, but was consistent only in 3 out of 10 PD + FOG 
subjects $(3 / 10=0.30)$. A comparison of the proportions of all subjects in each group who initiated walking with a consistent side

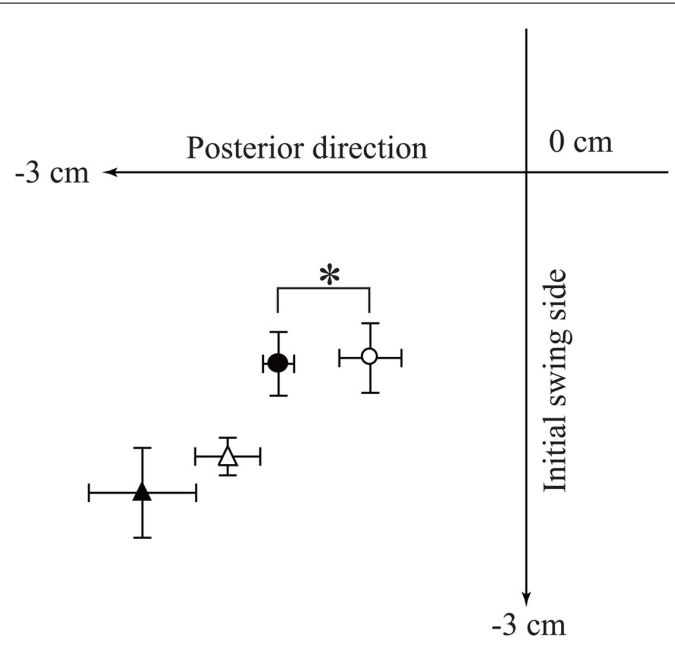

FIGURE 2 | First COP peak displacement during self-generated and cue-triggered gait initiation. Data points indicate the averages of the first COP peak displacements. Filled data points are from healthy controls, and open data points are from PD - FOG subjects. Circles indicate the data points during self-generated gait initiation, and triangles indicate the data points during cue-triggered gait initiation. An asterisk indicates a significant difference in COP peak displacement along the anteroposterior axis between the groups $(P<0.05)$. revealed significantly different proportions between the groups $(P<0.05)$. The first DLS/Cycle ratio, second COP peak displacement in the anteroposterior axis, and medial deviation of the COP from the second heel contact position were the parameters which showed significant differences between healthy controls and PD + FOG subjects $(P<0.05)$ and no significant differences between healthy controls and PD - FOG subjects. Effect sizes ranged from 1.70 to 2.02 in these parameters. For these parameters, the first DLS/Cycle ratio significantly correlated with FOGQ item 5 in PD + FOG $(P<0.05)$.

\section{DISCUSSION}

TRIAL-TO-TRIAL VARIABILITY OF THE INITIAL SWING SIDE

The initial swing side of gait initiation was consistent throughout the trials in most healthy controls and in most PD - FOG subjects. The finding in healthy controls was consistent with the finding in the previous study, but the finding in PD - FOG was inconsistent with the finding in PD + FOG in the previous study; the initial swing side was consistent in only 3 out of $10 \mathrm{PD}+$ FOG subjects (Okada et al., 2011). Accordingly, trial-to-trial variability of the initial swing side during gait initiation may be an abnormality specifically related to FOG. The variability of the motor pattern during steady-state gait in PD + FOG was found to be larger than that in PD - FOG (Hausdorff et al., 2003), indicating that a variable motor pattern may result in the variable initial swing side of gait initiation. One possible reason for this finding is the disease severity associated with FOG severity. It has been reported

Table 3 | Center of pressures and heel contact positions.

\begin{tabular}{|c|c|c|c|c|c|c|}
\hline & \multicolumn{2}{|c|}{ Cue-triggered } & \multicolumn{3}{|c|}{ Self-generated } & \multirow[t]{2}{*}{ Effect size } \\
\hline & Healthy & PD - FOG & Healthy & PD - FOG & PD + FOG & \\
\hline \multicolumn{7}{|c|}{ ANTEROPOSTERIOR COP PEAK DISPLACEMENT } \\
\hline Peak1 & $-2.7(0.4)$ & $-2.1(0.2)$ & $-1.8(0.1)$ & $-1.1(0.2)^{*}$ & $-1.1(0.2)^{*}$ & 0.00 \\
\hline Peak2 & $-2.7(0.4)$ & $-2.9(0.8)$ & $-2.5(0.4)$ & $-2.7(0.8)$ & $1.4(0.7)^{*}$ & 2.02 \\
\hline Peak3 & $56.7(1.2)$ & $48.1(3.2)^{*}$ & $57.2(0.8)$ & $48.1(3.2)^{*}$ & $39.9(3.8)^{*}$ & -0.96 \\
\hline \multicolumn{7}{|c|}{ MEDIOLATERAL COP PEAK DISPLACEMENT } \\
\hline Peak1 & $-2.20(0.31)$ & $-1.95(0.13)$ & $-1.31(0.22)$ & $-1.27(0.24)$ & $-1.14(0.23)$ & 0.21 \\
\hline Peak2 & $6.63(0.76)$ & $6.52(0.64)$ & $6.72(0.63)$ & $6.51(0.71)$ & $6.93(0.45)$ & 0.22 \\
\hline Peak3 & $-6.47(0.74)$ & $-9.99(0.94)^{*}$ & $-6.81(0.71)$ & $-9.67(1.06)^{*}$ & $-8.35(0.43)^{*}$ & 0.47 \\
\hline Peak4 & $9.78(1.19)$ & $6.83(1.10)$ & $8.25(0.62)$ & $6.83(0.94)$ & $5.88(0.66)$ & -0.38 \\
\hline \multicolumn{7}{|c|}{ MEDIAL DEVIATION OF COP FROM HEEL CONTACT POSITION } \\
\hline $1 s t$ & $0.34(0.19)$ & $1.15(0.51)$ & $0.23(0.20)$ & $1.40(0.39)^{*}$ & $1.93(0.57)^{*^{\dagger}}$ & 0.51 \\
\hline 2nd & $0.02(0.17)$ & $0.51(0.25)$ & $0.12(0.25)$ & $0.43(0.30)$ & $1.77(0.58)^{*}$ & 1.70 \\
\hline $3 r d$ & $-0.05(0.24)$ & $0.39(0.40)$ & $-0.12(0.20)$ & $0.67(0.33)$ & $1.04(0.48)$ & 0.43 \\
\hline
\end{tabular}

Mean (SE of mean).

Asterisks indicate significant difference between PD patients and Healthy controls.

Daggers indicate significant correlation between the parameter in PD+FOG and FOGQ item 5.

The data in PD + FOG were cited from the previous study (Okada et al., 2011). 


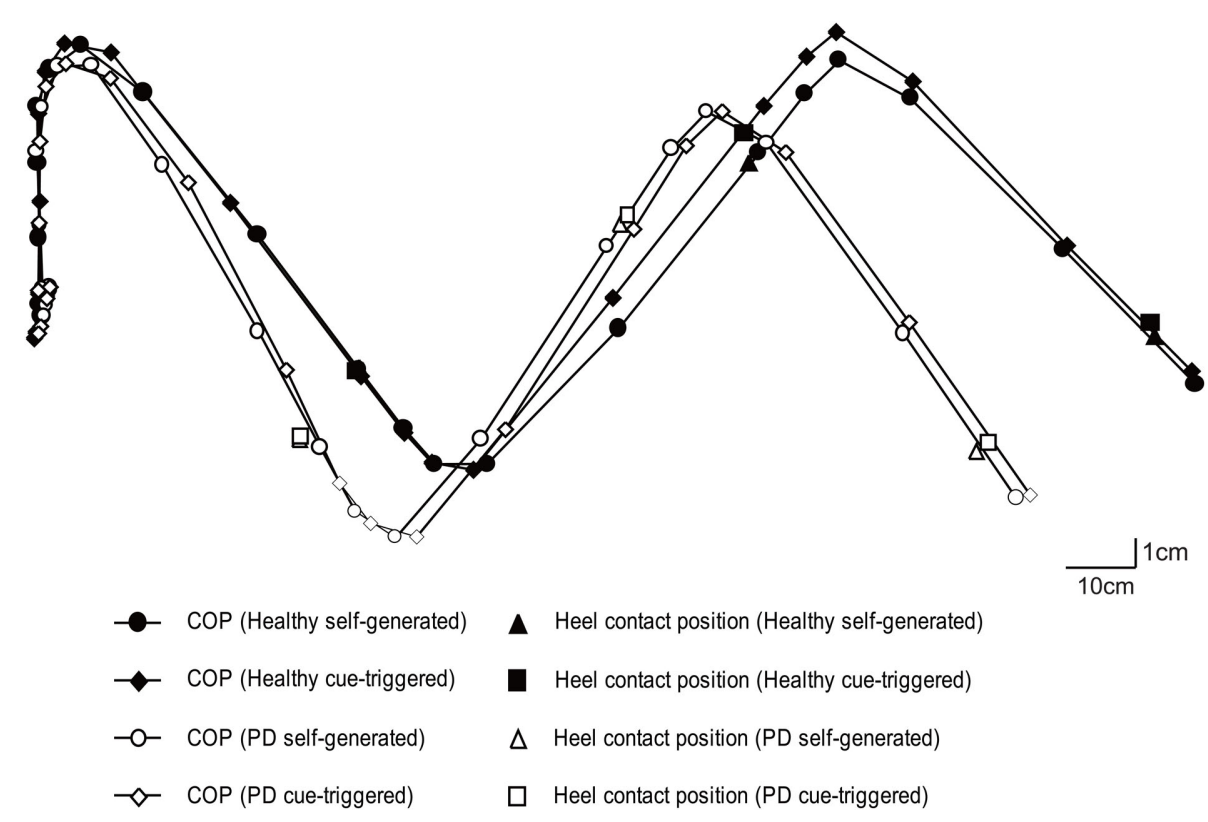

FIGURE 3 | Overall average of COP and heel contact position. The data points indicate the overall average of the COPs for each $5 \%$ bin of the total duration. The COP trajectories are depicted by lines connecting the nearest two COP data points.

that FOG severity partially depends on disease severity (Lamberti et al., 1997; Giladi et al., 2000, 2009; Chee et al., 2009). However, the disease severity was not statistically different between PD + FOG in the previous study (Okada et al., 2011) and PD - FOG in the present study, indicating that the variable initial swing side of gait initiation specifically present in PD + FOG is not related to the severity of the disease.

\section{ABNORMALITIES OF THE POSTURAL PHASE}

The first COP peak displacement along the anteroposterior axis, which reflects backward deviation of the COP during the postural phase, was found to be decreased during self-generated gait initiation in PD - FOG, which is consistent with the finding in previous studies that the posterior deviation of the COP during the postural phase is decreased in PD patients (Halliday et al., 1998; Dibble et al., 2004; Carpinella et al., 2007; Hass et al., 2008). Furthermore, this finding was consistent with the finding in PD + FOG (Okada et al., 2011). Therefore, the decreased first COP peak displacement along the anteroposterior axis in PD + FOG likely reflects the abnormality of the postural phase of self-generated gait initiation in PD patients in general, but is not specifically related to FOG.

\section{ABNORMALITIES OF THE OTHER PARAMETERS}

The first step time was prolonged in PD - FOG. The first step time was prolonged in PD + FOG as well (Okada et al., 2011). Therefore, a prolonged first step time is a parameter that is observable in the whole population of PD patients. The first step time defined in the present study involves not only the postural phase but also the first swing phase (Carpinella et al., 2007). The postural phase (Gantchev et al., 1996; Rosin et al., 1997; Halliday et al., 1998) and the first swing phase (Carpinella et al., 2007) are prolonged in PD patients, suggesting that the prolonged first step time in the present study reflects prolongation of the postural and first swing phases.

Step length and step velocity were decreased during selfgenerated gait initiation in PD - FOG. This finding was consistent with the previous findings that step length and step velocity are decreased during the first step of gait initiation in PD patients (Gantchev et al., 1996; Halliday et al., 1998; Dibble et al., 2004). Step length and step velocity have been reportedly abnormal during the first three steps of gait initiation in PD + FOG (Okada et al., 2011), indicating that abnormalities of step length and step velocity are not specifically related to FOG. The finding concerning step length conflicts with the previous finding that the step length during treadmill walking or steady-state gait is abnormal in PD + FOG, but is not abnormal in PD - FOG (Chee et al., 2009; Nanhoe-Mahabier et al., 2011). It has been reported that PD + FOG decreases step length as the number of steps increases (Iansek et al., 2006; Chee et al., 2009). Gait initiation begins from standing, indicating that the number of steps executed before the beginning of gait initiation is zero. In contrast, steady-state gait begins after at least three steps of gait initiation (Mann et al., 1979). Thus, the number of steps executed before gait initiation is theoretically less than that before steady-state gait is achieved. Accordingly, the conflicting abnormalities of step length between gait initiation and steady-state gait may reflect a different sequential effect of the steps on FOG.

The third COP peak displacement and the first heel contact position significantly deviated to the initial swing side in $\mathrm{PD}-$ FOG. This finding is consistent with that in PD + FOG (Okada et al., 2011), indicating that this abnormality is not specifically related to FOG. It has been hypothesized that a small weight shift to the initial stance side followed by normal weight shifting to 
the initial swing side may be the cause of the excessive shifting of the COP to the initial swing side, leading to an increase in the third COP peak displacement in PD + FOG (Okada et al., 2011). However, the second COP peak displacement along the mediolateral axis was not prominently decreased in the present study. Therefore, the small weight shift toward the initial stance side should not be considered the cause of the excessive deviation of the COP toward the initial swing side in PD patients.

The medial deviation of the COP from the first heel contact position was increased in PD - FOG. This finding is consistent with the finding in PD + FOG (Okada et al., 2011), indicating that this parameter is not specifically related to FOG. It has been hypothesized that the increase in the medial deviation of the COP from the first heel contact position may be explained by the possibility that a certain amount of weight remains on the tracing leg contralateral to the heel contact side (Okada et al., 2011). If this is true, medial deviation of the COP from the heel contact position should have been associated with delayed heel off leading to prolonged DLS. However, the first DLS ratio was not prolonged in PD - FOG, indicating that prolonged DLS is likely not the major cause of the increase in the medial deviation of the COP from the first heel contact position in PD patients.

\section{ABNORMALITIES DURING CUE-TRIGGERED GAIT INITIATION}

An auditory external cue is considered to be effective if some parameters are abnormal during self-generated gait initiation but are not abnormal during cue-triggered gait initiation. The first step time, first step velocity, first COP peak displacement along the anteroposterior axis, second DLS ratio, and medial deviation of the COP from the first heel contact position were abnormal during self-generated gait initiation, but not during cue-triggered gait initiation. The first step time and first step velocity involve the postural phase and the first COP peak displacement occurs exactly during the postural phase. In contrast, the second DLS/Cycle ratio and medial deviation of the COP from the first heel contact position occur after the postural phase. Accordingly, the auditory external cue is effective for normalizing gait initiation not only during the postural phase but also after the postural phase of gait initiation in PD - FOG.

\section{ABNORMALITIES OF GAIT INITIATION SPECIFIC TO PD + FOG}

The present study compared the abnormalities during selfgenerated gait initiation in PD - FOG with those in PD + FOG cited from the previous study (Okada et al., 2011) by three procedures. First, the statistical significances of the abnormalities were compared between PD + FOG and PD - FOG. Second, the effect sizes between the means in PD + FOG and those in PD - FOG were calculated in order to estimate the magnitude of the difference in means between the groups. Third, the statistical significances of the correlation coefficients between the parameters of gait initiation and FOG severity were cited from the previous study (Okada et al., 2011). We considered that the parameters showing statistically significant correlations with FOG severity in PD + FOG, significant abnormality in PD + FOG, no significant abnormality in PD - FOG, and a large effect size between the groups were specifically related to FOG.
Medial deviation of the COP from the first heel contact position has been believed to be abnormal during gait initiation specifically in PD + FOG (Okada et al., 2011). However, the present study found that the medial deviation of the COP from the first heel contact position was abnormal in PD - FOG, indicating that abnormality of the medial deviation of the COP from the first heel contact position is not specifically related to FOG. The medial deviation of the COP from the second heel contact position was abnormal in PD + FOG, but not in those without FOG (Okada et al., 2011). However, the medial deviation of the COP from the second heel contact position was not significantly correlated with FOGQ item 5 (Okada et al., 2011). Accordingly, it is not sufficient to consider medial deviation of the COP from the second heel contact position to be a parameter specifically related to FOG.

The DLS/Cycle ratio was not significantly prolonged during the first step of gait initiation in PD - FOG, but was significantly prolonged in PD + FOG. The effect size in the first DLS/Cycle ratio between PD + FOG and PD - FOG was considered to be large according to classical classification (Cohen, 1992). Additionally, the first DLS/Cycle ratio was correlated with FOGQ item 5 (Okada et al., 2011). Therefore, the duration of DLS during first step of gait initiation is considered to be a parameter specifically related to FOG.

One possible question is whether the prolonged DLS during the first step of gait initiation in PD + FOG is an abnormality specifically for gait initiation. The prolonged DLS is present even during steady-state gait in PD patients (Koozekanani et al., 1987; Morris et al., 1996, 2005; Hausdorff et al., 1998; Chien et al., 2006). However, it has not been reported that the prolonged DLS during steady-state gait is specifically related to FOG. The prolonged DLS during the first step of gait initiation is likely to relate specifically to FOG during gait initiation rather than being a general manifestation of FOG during any state of gait, because no significant increase in the DLS/Cycle ratio was present after the first step of gait initiation.

A previous finding concerning steady-state gait in PD + FOG gives us some suggestions why the prolonged DLS during the first step of gait initiation is specifically related to FOG. Before freezing, the DLS phase is larger than that before stopping during walking in PD patients in an "OFF" state (Nieuwboer et al., 2001). These previous researchers hypothesized that the prolonged DLS is caused by postural instability, leading to compensation for the increase of the time during which both feet are in contact with the ground or an inability to adequately transfer weight as a preparation for stepping. The prolonged DLS during gait initiation found as a parameter specifically related to FOG in the present study may reflect such impairments. However, this previous finding does not explain why the first DLS is related to FOG. Further studies are needed to elucidate the mechanisms underlying this finding.

\section{CLINICAL IMPLICATION}

Improving gait start hesitation is an important goal of physical therapy for PD + FOG patients, but various approaches to this problem have been designed without sufficient understanding of FOG. The present study found that variability of the initial swing side is a specific manifestation of FOG during gait initiation. This finding may indicate that PD + FOG patients may be choosing 
the initial swing side immediately before gait initiation. This may increase the information process in the preparation of gait initiation in PD + FOG patients. If this hypothesis is true, one possible implication for physical therapy in $\mathrm{PD}+\mathrm{FOG}$ patients is that patients could be instructed to choose the initial swing side of gait initiation in advance. In addition, the prolonged DLS in the first step of gait initiation was found to be abnormal in PD + FOG. The prolonged DLS means that the first toe off of the initial stance leg is delayed. One possible implication for physical therapy in $\mathrm{PD}+$ FOG patients based on this finding is emphasizing weight shift to the initial swing side in the early phase of gait initiation by oral instruction or by manual handling of the body, to assist the first toe off of the initial stance leg.

\section{LIMITATION}

We cannot rule out the effect of antiparkinsonian medications on abnormalities of gait initiation specifically present in PD + FOG.

\section{REFERENCES}

Bartels, A. L., Balash, Y., Gurevich, T., Schaafsma, J. D., Hausdorff, J. M., and Giladi, N. (2003). Relationship between freezing of gait (FOG) and other features of Parkinson's: FOG is not correlated with bradykinesia. J. Clin. Neurosci. 10, 584-588.

Burleigh-Jacobs, A., Horak, F. B., Nutt, J. G., and Obeso, J. A. (1997). Step initiation in Parkinson's disease: influence of levodopa and external sensory triggers. Mov. Disord. 12, 206-215.

Carpinella, I., Crenna, P., Calabrese, E., Rabuffetti, M., Mazzoleni, P., Nemni, R., and Ferrarin, M. (2007). Locomotor function in the early stage of Parkinson's disease. IEEE Trans. Neural Syst. Rehabil. Eng. 15, 543-551.

Chee, R., Murphy, A., Danoudis, M., Georgiou-Karistianis, N., and Iansek, R. (2009). Gait freezing in Parkinson's disease and the stride length sequence effect interaction. Brain 132, 2151-2160.

Chien, S. L., Lin, S. Z., Liang, C. C., Soong, Y. S., Lin, S. H., Hsin, Y. L., Lee, C. W., and Chen, S. Y. (2006). The efficacy of quantitative gait analysis by the GAITRite system in evaluation of parkinsonian bradykinesia. Parkinsonism Relat. Disord. 12, 438-442.

Cohen, J. (1992). A power primer. Psychol. Bull. 112, 155-159.

Coren, S. (1993). The lateral preference inventory for measurement of handedness, footedness, eyedness and earedness: norms for young adults. Bull. Psychon. Soc. 31, 1-3.

Crenna, P., Frigo, C., Giovannini, P., and Piccolo, I. (1990). "The initiation of gait in Parkinson's disease," in Motor Disturbances II, ed.
C. D. Marsden (New York: Academic Press), 161-173.

Cupp, E. W., Duke, B. O., Mackenzie, C. D., Guzmán, J. R., Vieira, J. C., Mendez-Galvan, J., Castro, J., Richards, F., Sauerbrey, M., Dominguez, A., Eversole, R. R., and Cupp, M. S. (2004). The effects of long-term community level treatment with ivermectin (Mectizan) on adult Onchocerca volvulus in Latin America. Am. J. Trop. Med. Hyg. 71, 602-607.

Dibble, L. E., Nicholson, D. E., Shultz, B., Williams, B. A., Marcus, R. L., and Moncur, C. (2004). Sensory cueing effects on maximal speed gait initiation in persons with Parkinson's disease and healthy elders. Gait Posture 19, 215-225.

Gantchev, N., Viallet, F., Aurenty, R., and Massion, J. (1996). Impairment of posturo-kinetic co-ordination during initiation of forward oriented stepping movements in parkinsonian patients. Electroencephalogr. Clin. Neurophysiol. 101, 110-120.

Giladi, N., Shabtai, H., Simon, E. S., Biran, S., Tal, J., and Korczyn, A. D. (2000). Construction of freezing of gait questionnaire for patients with Parkinsonism. Parkinsonism Relat. Disord. 6, 165-170.

Giladi, N., Tal, J., Azulay, T., Rascol, O., Brooks, D. J., Melamed, E., Oertel, W., Poewe, W. H., Stocchi, F., and Tolosa, E. (2009). Validation of the freezing of gait questionnaire in patients with Parkinson's disease. Mov. Disord. 24, 655-661.

Halliday, S. E., Winter, D. A., Frank, J. S., Patla, A. E., and Prince, F. (1998). The initiation of gait in young, elderly, and Parkinson's disease subjects. Gait Posture 8, 8-14.

Hass, C. J., Gregor, R. J., Waddell, D. E., Oliver, A., Smith, D. W., Fleming, R.

LED in PD - FOG was significantly higher than that in PD + FOG. Levodopa improves FOG (Bartels et al., 2003; Schaafsma et al., 2003). In the present study involving PD - FOG subjects and in the previous study involving PD + FOG subjects, the experiment was conducted when PD patients were in an "ON" state. Therefore, it is possible that the fact that some gait parameters which did not reveal statistically significant abnormalities in PD - FOG but did reveal significant abnormalities in PD + FOG may be partially related to the effect of antiparkinsonian medications.

\section{CONCLUSION}

The first three steps of gait initiation are abnormal in PD - FOG. An external cue normalizes some abnormalities not only during the postural phase but also after the postural phase of gait initiation. Prolonged DLS during the first step and the trial-to-trial variability of the initial swing side are likely to be abnormalities specifically related to FOG.

P., and Wolf, S. L. (2004). The influence of Tai Chi training on the center of pressure trajectory during gait initiation in older adults. Arch. Phys. Med. Rehabil. 85, 1593-1598.

Hass, C. J., Waddell, D. E., Wolf, S. L., Juncos, J. L., and Gregor, R. J. (2008). Gait initiation in older adults with postural instability. Clin. Biomech. (Bristol, Avon) 23, 743-753.

Hausdorff, J. M., Cudkowicz, M. E., Irtion, R., Wei, J. Y., and Goldberger, A. L. (1998). Gait variability and basal ganglia disorders: stride-tostride variations of gait cycle timing in Parkinson's disease and Huntington's disease. Mov. Disord. 13, 428-437.

Hausdorff, J. M., Schaafsma, J. D., Balash, Y., Bartels, A. L., Gurevich, T., and Giladi, N. (2003). Impaired regulation of stride variability in Parkinson's disease subjects with freezing of gait. Exp. Brain Res. 149, 187-194.

Hiraoka, K., Matuo, Y., Iwata, A., Onishi, T., and Abe, K. (2006). The effects of external cues on ankle control during gait initiation in Parkinson's disease. Parkinsonism Relat. Disord. 12 97-102.

Iansek, R., Huxham, F., and McGinley, J. (2006). The sequence effect and gait festination in Parkinson disease: contributors to freezing of gait? Mov. Disord. 21, 1419-1424.

Koozekanani, S. H., Balmaseda, M. T., Fatehi, M. T., and Lowney, E. D. (1987). Ground reaction forces during ambulation in parkinsonism: pilot study. Arch. Phys. Med. Rehabil. 68, 28-30.

Lamberti, P., Armenise, S., Castaldo, V., de Mari, M., Iliceto, G., Tronci, P., and Serlenga, L. (1997). Freezing gait in Parkinson's disease. Eur. Neurol. 38, 297-301.
Lee, J. J., and Tseng, C. (2001).Uniform power method for sample size calculation in historical control studies with binary response. Control. Clin. Trials 22, 390-400.

Mann, R. A., Hagy, J. L., White, V., and Liddell, D. (1979). The initiation of gait. J. Bone Joint Surg. Am. 61, 232-239.

Meng, W., Dong, Y., Liu, J., Wang, Z., Zhong, X., Chen, R., Zhou, H., Lin, M., Jiang, L., Gao, F., Xu, T., Chen, Q., and Zeng, X. (2009). A clinical evaluation of amlexanox oral adhesive pellicles in the treatment of recurrent aphthous stomatitis and comparison with amlexanox oral tablets: a randomized, placebo controlled, blinded, multicenter clinical trial. Trials 10, 30.

Morris, M. E., Iansek, R., Matyas, T. A., and Summers, J. J. (1996). Stride length regulation in Parkinson's disease. Normalization strategies and underlying mechanisms. Brain 119, 551-568.

Morris, M. E., Iansek, R., McGinlev, J., Matyas, T., and Huxham, F. (2005). Three-dimensional gait biomechanics in Parkinson's disease: evidence for a centrally mediated amplitude regulation disorder. Mov. Disord. 20, 40-50.

Nanhoe-Mahabier, A., Snijders, A. H., Delval, A., Weerdesteyn, V., Duysens, J., Overeem, S., and Bloem, B. R. (2011). Walking patterns in Parkinson's disease with and without freezing of gait. Neuroscience 182, 217-224.

Nieuwboer, A., Dom, R., De Weerdt, W., Desloovere, K., Fieuws, S., and Broens-Kaucsik, E. (2001). Abnormalities of the spatiotemporal characteristics of gait at the onset of freezing in Parkinson's disease. Mov. Disord. 16, 1066-1075. 
Nieuwboer, A., Dom, R., De Weerdt, W., Desloovere, K., Janssens, L., and Stijin, V. (2004). Electromyographic profiles of gait prior to onset of freezing episodes in patients with Parkinson's disease. Brain 127, 1650-1660.

Okada, Y., Fukumoto, T., Takatori, K., Nagino, K., and Hiraoka, K. (2011). Abnormalities of the first three steps of gait initiation in patients with Parkinson's disease with freezing of gait. Parkinsons Dis. 202937.

Plotnik, M., Giladi, N., Balash, Y., Peretz, C., and Hausdorff, J. M. (2005). Is freezing of gait in Parkinson's disease related to asymmetric motor function? Ann. Neurol. 57, 656-663.
Plotnik, M., Giladi, N., and Hausdorff, J. M. (2008). Bilateral coordination of walking and freezing of gait in Parkinson's disease. Eur. J. Neurosci. 27, 1999-2006.

Rosin, R., Topka, H., and Dichgans, J. (1997). Gait initiation in Parkinson's disease. Mov. Disord. 12, 682-690.

Sacks, H., Chalmers, T. C., and Smith, H. Jr. (1982). Randomized versus historical controls for clinical trial. Am. J. Med. 72, 233-240.

Schaafsma, J. D., Balash, Y., Gurevich, T., Bartels, A. L., Hausdorff, J. M., and Giladi, N. (2003). Characterization of freezing of gait subtypes and the response of each to levodopa in Parkinson's disease. Eur. J. Neurol. 10, 391-398.
Thomas, J. R., French, K. E., and Humphries, C. A. (1986). The use of meta-analysis in exercise and sport: a tutorial. Res. Q. Exerc. Sport 57, 196-204.

Conflict of Interest Statement: The authors declare that the research was conducted in the absence of any commercial or financial relationships that could be construed as a potential conflict of interest.

Received: 25 September 2011; accepted: 06 December 2011; published online: 29 December 2011.

Citation: Okada Y, Fukumoto T, Takatori K, Nagino K and Hiraoka K (2011)
Variable initial swing side and prolonged double limb support represent abnormalities of the first three steps of gait initiation in patients with Parkinson's disease with freezing of gait. Front. Neur. 2:85. doi: 10.3389/fneur.2011.00085

This article was submitted to Frontiers in Movement Disorders, a specialty of Frontiers in Neurology.

Copyright (C) 2011 Okada, Fukumoto, Takatori, Nagino and Hiraoka. This is an open-access article distributed under the terms of the Creative Commons Attribution Non Commercial License, which permits non-commercial use, distribution, and reproduction in other forums, provided the original authors and source are credited. 